\title{
DEVELOPMENT OF SOFTCORE PROCESSOR
}

\author{
Mohammed Zaheer ${ }^{1}$, A.M Khan ${ }^{2}$ \\ ${ }^{1}$ Research Student Department of Electronics Mangalore University, Karnataka, India \\ ${ }^{2}$ proffesor chairman, Department of Electronics Mangalore University, Karnataka, India
}

\begin{abstract}
Designing of softcore processor on FPGA is based on software hardware co design. A soft-core processor is a hardware description language (HDL) model of a specific processor (CPU) that can be customized for a given application and synthesized for an ASIC or FPGA target. Embedded Systems which performs specific application using components having hardware and software together is known as embedded system. With hardware design getting complicated day by day, using of software tools for designing the system and testing has made the necessity of today's technology. Due to the reduced size and greater complexity of hardware models, it requires a higher technological tool for simulation to satisfy the needs of the designers. The more efficient the simulator, complexity in the hardware model can be easily executed in small intervals of time, resulting in design product in a stipulated time intervals.
\end{abstract}

The design of soft core processor system involves strict performance involving area, time, power, cost constraints. Advantages of Softcore processor design improves in design quality reduce integration and test time, supporting growing complexity of embedded system, processor cores, high level hardware synthesizing capabilities, ASIC/FPGA development and platform independence.

Xilinx microblaze softcore processor support package allows to create mixed Hardware Software application using micro blaze processor. These processors are called as soft-core processor. Soft IP core of Xilinx micro blaze can be used on series of FPGA.

Keywords: soft-core processor, Xilinx, embedded system $* * *$

\section{INTRODUCTION}

Embedded Systems are hardware and software components working together to perform a specific application. They are used in many applications and play an important role in our day to day life. They can be found in our automobiles, in the medical field, in industrial control systems, and in entertainment electronics. The hardware platform of the embedded system often consists of a microprocessor, in build memory, an output display, an input device for a user to enter data and application software. The embedded systems are becoming increasingly complex which makes its design complex. The constraints in terms of area usage, size, power consumption and performance further add to this complexity. In order to reduce the amount of time spent on development as well as debugging of soft core processor, hardware/software co-design methodology is currently preferred. Designing each and every hardware component is not a practical proposition while designing a complex embedded system and in that case even the cost will be prohibitively high. So a more practical proposition is the usage of components which are designed and tested before hand in the form of intellectual property (IP). They can be synthesized on Field Programmable Gate Array (FPGA), therefore pre-designed and pre-tested intellectual property (IP) cores provide designers with lot of flexibility. FPGA vendors are providing with configurable soft-core processor that can be synthesized on FPGAs. FPGAs with soft cores processor provide designers with increased flexibility and debugging. The innovations in technology of
Programmable logic design have lead to FPGAs. These FPGAs support implementation of full computer system in a single FPGA chip, soft core processor being a vital component of such a system.

\section{SOFT-CORE PROCESSORS}

A soft-core processor is a hardware description language (HDL) model of a specific processor (CPU) that can be customized for a given application and synthesized on ASIC or FPGA target [1]. The soft core processors have the advantages of flexibility and ease of customization. Technology independent nature of soft processors allows it to be synthesized for any given target. These targets in terms of ASIC and FPGA technologies make them immune to safety considerations much better than circuit or logic based technologies. In addition, the higher level of abstraction incorporated in soft core processors using HDL in the architecture of soft core processor improves its overall design. We are aware oof the increasingly common usage of sort core processors in commercially available forms. Examples of soft processor cores are: Altera Lattice Micro 32, Nios / Nios II and Xilinx Pico Blaze Micro Blaze. These soft-core processor offer logic elements and memory with Intellectual Property (IP) for the development of system-on programmable - chip (SOPC). A complete microprocessor system can be developed using MicroBlaze processor, Xilinx as it provides the different cores needed to develop such as system. Interrupt controllers, memory controllers, Ethernet controllers, UARTs (UART - Universal 
Asynchronous Receiver/Transmitter), timers, buses, and others remain the essential part cores. FPGA is a very versatile unit which can house all the cores on the on-chip memory. The concept of placing this entire essential component on a single chip refers to programmable chip (SOPC) [2]. Some of the components in the form of intellectual property cores are reusable. An IP core is a standard block of logic or data that can be used to build a larger or more complex system. Various factors affect the design of IP cores, major factors being level of optimization and flexibility of re-use. Based on these factors IP cores are classified into three categories, viz., soft cores, firm cores, and hard cores [6].

\section{HARDWARE PLATFORM FOR SOFTCORE}

The advancement in technology has made computer systems progressively smaller. The stage has reached where a compete computer system can be implemented on a single chip using Field Programmable Gate Array (FPGA) technology. An integral part of such is system is a soft core processor. A soft-core processor is a microprocessor which is defined in software which can be synthesized in programmable hardware; FPGAs allow repeated programming and reprogramming making using of different algorithms. C-based design flows allow FPGA to be programmed easily. A major problem faced by the embedded system designers is verification of the systems. This is overcome by C-like code having a design flow of a model to check the implemented subsystem. The hardware consists of different components which are connected to soft-core processor. As shown in fig.1, [3] the various components in the hardware are GPIO push buttons, GPIO LEDS, GPIODIP Switch. The multi featured FPGA and onchip collection of peripherals including 128MByte, HDMI Video, Gbit Ethernet, 16-bit DDR2 memory, audio ports and USB make the Atlys board suitable for designing of digital systems, which includes Xilinx's MicroBlaze embedded processor designs.

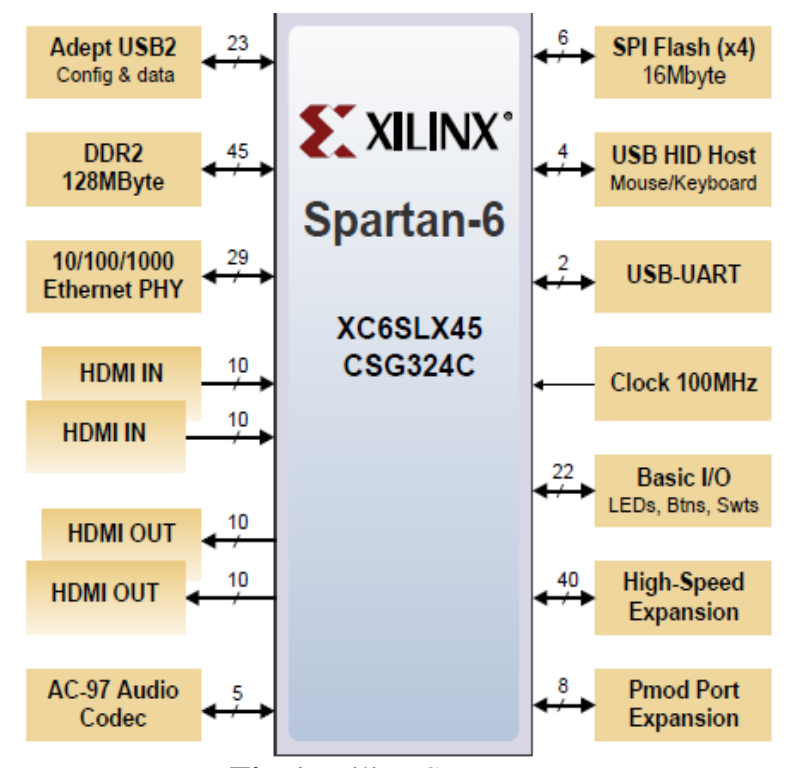

Fig-1: Xilinx Spartan -6

\section{DESIGN OF SOFTCORE PROCESSOR}

There are various tools for development of soft core processors, including Altera Nios / Nios II, Lattice Micro 32, and Xilinx Micro Blaze. These tools support quick development of systems on programmable chip by incorporating memory and logic elements as well as several intellectual Property (IP).There is are many soft core processors in the market, MicroBlaze softcore processor by Xilinx being a popular one, developed by Xilinx, specialized in development of FPGAs. Moreover, they also offer soft IP cores that target their FPGAs. fig.2, [4] MircroBlaze processors are essentially 32 bit processors optimized for embedded applications. The typical speed at which they operate are upto $200 \mathrm{MHz}$. These processors are based on Harward RISC architecture which process 32 bit instructions. The processing of information is through a 3 stage pipleline, 32 bit register, shift unit and two level interrupt. The storage memory can be an external peripheral or an internal chip. Both data and program are stored in local memory which makes use of a Block RAM. The size of the local memory may from $4 \mathrm{kB}$ to $64 \mathrm{kB}$. Local Memory Bus (LMB) and the LMB BRAM Interface Controllers are used to connect local memory to MicroBlaze. In addition, another type of interface, known as On-chip Peripheral Bus (OPB) can be used for interfacing Chip with memory, either on a chip or off-chip.

There is a need to connect Xilinx microprocessor debugger with MicroBlaze Debug logic which is implemented through MicroBlaze Debug module. Xilinx microprocessor debugger can be used for downloading software, it provides provision to set break points and independently view register and memory content. Development of embedded system using soft core processor needs a set of IP cores. Xilinx offers the Embedded Development Kit (EDK) which includes Xilinx Platform studio for meeting this requirement.

\section{IMPLEMENTATION OF HARDWARE AND SOFTWARE PLATFORM}

Hardware software co-design is a reunion of system level objectives utilizing the hardware and software in a system through their concurrent design. The method of hardware design can be implemented on different series of FPGA. The software design that is build using the embedded development kit available in Xilinx tool ISE design suite 14.1.Designing a soft core processor is versatile task due enormous flexibility available to the designer. The Xilinx platform studio offers this flexibility to the designer. This soft-core processor is the core processor which is implemented on the hardware of Xilinx FPGA families according of to the peripherals required by the designer. It is an ideal downloadable solution for FPGA design offering $\mathrm{C}$, $\mathrm{C}++$ compiler which converts into HDL synthesis and simulation, while implementation. The figure 3 [4] describes the implementation of hardware and software platform. The embedded hardware is developed by a system targeting a board using Base System Builder Wizard. This Extend the hardware system, by adding peripherals from the fixed IP 
core. This Generate HDL Netlists using PlatGen. To Create and debug the softwareapplication using Software Development Kit (SDK), debugging is done using Xilinx Microprocessor Debug (XMD) and the GNU debugger (gdb).Finally it is deployed in hardware by Generating the bit stream and configure the FPGA using iMPACT.

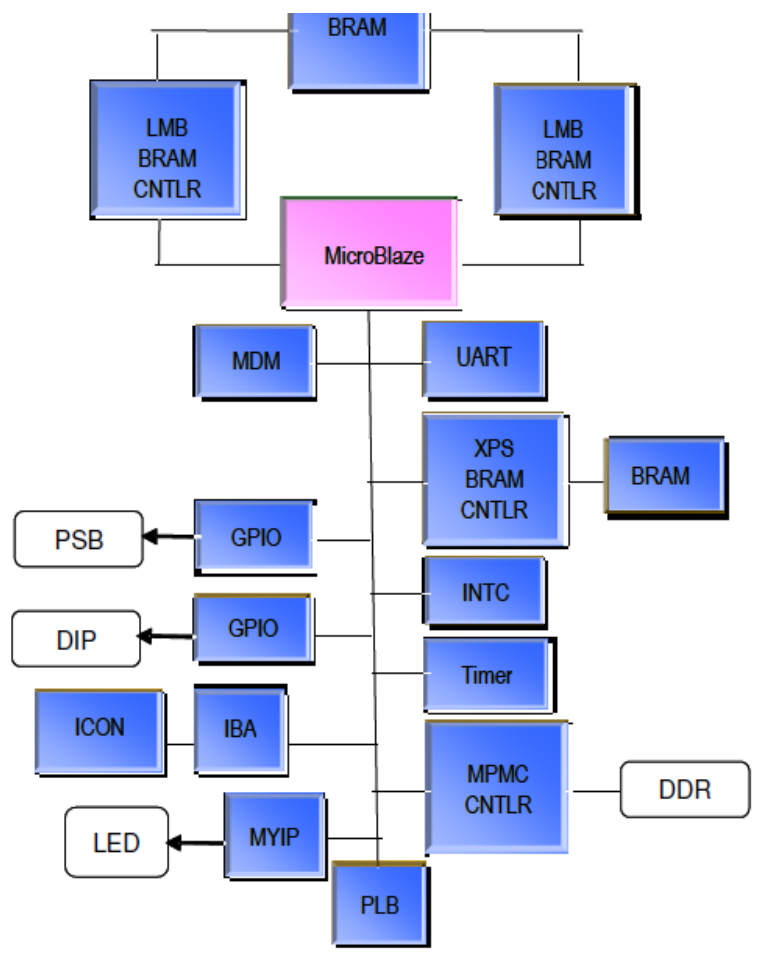

Fig 2: Peripherals of Micro Blaze

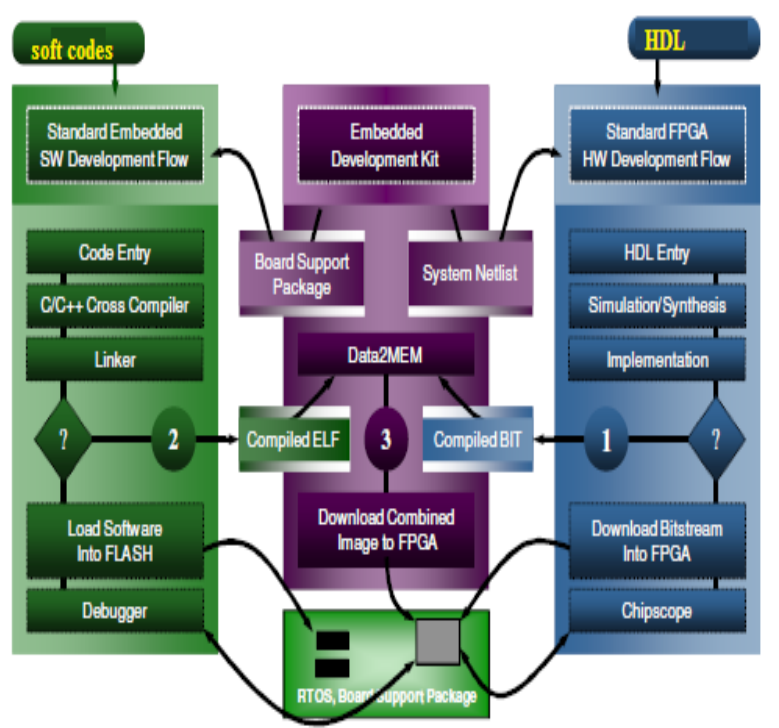

Fig 3: Development of soft core processor

\section{CONCLUSION}

The complexity and sophistication that occurs in independent hardware and software design can be overcome by mixed design of hardware and software. The versatility and reach of embedded system for diverse applications makes its design in today's technology enabled world and implementing it through hardware-software co-design has emerged as an attractive option for a specific application according to the designer's requirement.

\section{REFERENCES}

[1]. Jason G. Tong, Ian D. L. Anderson and Mohammed A. S. Khalid. "Soft-Core Processors for Embedded Systems" The 18th International Conference on Microelectronics (ICM) 2006 University of Winds or Department of Electrical and Computer Engineering.

[2]. Franjo Plavec,"Soft-Core Processor Design" Thesis Department Of Electrical And Computer Engineering University Of Toronto, 2004.

[3]. Atllys ${ }^{\mathrm{TM}}$ Board Reference Manual

[4]. Xilinx Incorporated Website, www.xilinx.com

[5]. Wagdy H Mahmoud "Soft Core and Hard Core Processors", () American Society for Engineering Education, 2009 University of the District of Columbia, Incorporating.

[6]. R. K. Gupta and Y. Zorian, "Introducing Core-Based System Design," IEEE Design and Test of Computers, vol. 14, no. 4 (October-December 1997), pp 15-25. 